\title{
Pin1 Modulates the Synaptic Content of NMDA Receptors via Prolyl-Isomerization of PSD-95
}

\author{
Roberta Antonelli, ${ }^{1}$-Roberto De Filippo, ${ }^{1}$ Silvia Middei, ${ }^{2}$ Stefka Stancheva, ${ }^{3}$ Beatrice Pastore, ${ }^{1}$ \\ Martine Ammassari-Teule, ${ }^{2}$ Andrea Barberis, ${ }^{3}$ Enrico Cherubini, ${ }^{1,4}$ and Paola Zacchi ${ }^{1,4}$ \\ ${ }^{1}$ International School for Advanced Studies, 34136 Trieste, Italy, ${ }^{2}$ National Research Council Institute of Cell Biology and Neurobiology, Santa Lucia \\ Foundation, 00143 Rome, Italy, ${ }^{3}$ Istituto Italiano di Tecnologia, 16163 Genoa, Italy, and ${ }^{4}$ European Brain Research Institute, 00143 Rome, Italy
}

Phosphorylation of serine/threonine residues preceding a proline regulates the fate of its targets through postphosphorylation conformational changes catalyzed by the peptidyl-prolyl cis-/trans isomerase Pin1. By flipping the substrate between two different functional conformations, this enzyme exerts a fine-tuning of phosphorylation signals. Pin1 has been detected in dendritic spines and shafts where it regulates protein synthesis required to sustain the late phase of long-term potentiation (LTP). Here, we demonstrate that Pinl residing in postsynaptic structures can interact with postsynaptic density protein-95 (PSD-95), a key scaffold protein that anchors NMDA receptors (NMDARs) in PSD via GluN2-type receptor subunits. Pin1 recruitment by PSD-95 occurs at specific serine-threonine/proline consensus motifs localized in the linker region connecting PDZ2 to PDZ3 domains. Upon binding, Pin1 triggers structural changes in PSD-95, thus negatively affecting its ability to interact with NMDARs. In electrophysiological experiments, larger NMDA-mediated synaptic currents, evoked in CA1 principal cells by Schaffer collateral stimulation, were detected in hippocampal slices obtained from Pin $1^{-/-}$ mice compared with controls. Similar results were obtained in cultured hippocampal cells expressing a PSD-95 mutant unable to undergo prolyl-isomerization, thus indicating that the action of Pin1 on PSD-95 is critical for this effect. In addition, an enhancement in spine density and size was detected in CA1 principal cells of Pin ${ }^{-1-}$ or in Thy-1GFP mice treated with the pharmacological inhibitor of Pin1 catalytic activity PiB.

Our data indicate that Pin 1 controls synaptic content of NMDARs via PSD-95 prolyl-isomerization and the expression of dendritic spines, both required for LTP maintenance.

Key words: dendritic spines; glutamatergic transmission; hippocampus; NMDA receptors; Pin1; PSD-95

Significance Statement

PSD-95, a membrane-associated guanylate kinase, is the major scaffolding protein at excitatory postsynaptic densities and a potent regulator of synaptic strength and plasticity. The activity of PSD-95 is tightly controlled by several post-translational mechanisms including proline-directed phosphorylation. This signaling cascade regulates the fate of its targets through postphosphorylation conformational modifications catalyzed by the peptidyl-prolyl cis-/trans isomerase Pin1. Here, we uncover a new role of Pin1 in glutamatergic signaling. By interacting with PSD-95, Pin1 dampens PSD-95 ability to complex with NMDARs, thus negatively affecting NMDAR signaling and spine morphology. Our findings further emphasize the emerging role of Pin1 as a key modulator of synaptic transmission.

\section{Introduction}

PSD-95 represents a major core scaffolding protein enriched at PSDs of excitatory synapses (Chen et al., 2005), which regulates several aspects of synapse dynamics, from synapse maturation to synaptic strength and plasticity (Kim and Sheng, 2004; Elias and Nicoll, 2007; Xu, 2011). This scaffolding molecule is one of the 
earliest proteins detected in the PSD (Rao et al., 1998) and regulation of its synaptic clustering is essential for proper synapse formation and maturation. PSD-95 shares with other membrane-associated guanylate kinase (MAGUK)-family members a multimodular structure composed of three PDZ (PSD-95/ Discs large/zona occludens-1) domains, a Src homology 3 (SH3) domain and a catalytically inactive guanylate kinase domain (Kim and Sheng, 2004). This structural architecture allows anchoring NMDA and AMPA types of glutamate receptors at postsynapses, and tethering them to intracellular signaling complexes and cytoskeletal elements responsible for their dynamic changes (Opazo et al., 2012).

Several post-translational modifications have been shown to control the dynamic deposition of PSD-95 at synapses, such as palmitoylation (Craven et al., 1999), serine/threonine phosphorylation (Morabito et al., 2004; Gardoni et al., 2006; Kim et al., 2007) and tyrosine phosphorylation (Du et al., 2009). Phosphorylation on certain serine or threonine residues preceding a proline regulates the fate of its targets through postphosphorylation conformational modifications catalyzed by the peptidyl-prolyl cis-/trans isomerase (PPIase) Pin1 (Lu et al., 2007). This enzyme acts by flipping the substrate between two different conformations that are functionally diverse, thus exerting a fine-tuning of phosphorylation signals (Liou et al., 2011). At inhibitory synapses, Pin1-dependent isomerization of gephyrin, the functional homolog of PSD-95 at inhibitory synapses, affects GlyRs function (Zita et al., 2007). At GABAergic synapses, Pin 1 recruitment by the cell adhesion molecule neuroligin 2 negatively modulates its ability to complex with gephyrin, leading to a downregulation of GABAergic transmission (Antonelli et al., 2014). At glutamatergic synapses Pin 1 has been detected in dendritic shafts and spines where it acts by suppressing protein synthesis required to sustain the late phase of long-term potentiation (LTP; Westmark et al., 2010).

The high abundance of Pin 1 at excitatory synaptic contacts and the observation that PSD-95 bears potential recognition sites for prolyl-isomerization prompted us to investigate whether such MAGUK member may undergo postphosphorylation modulation of its activity. Here, we provide evidence that endogenous PSD-95 can recruit Pin 1 at consensus motifs located between the second and third PDZ domains. We show that postphosphorylation prolyl-isomerization negatively regulates the ability of PSD-95 to complex with NMDA receptors (NMDARs), leading to a downregulation of the NMDAR-mediated synaptic transmission associated with a decrease in dendritic spines density.

\section{Materials and Methods}

Constructs. GFP-tagged PSD-95and FLAG-tagged GluN2B constructs were kindly provided by Dr. Vicini (Georgetown University School of Medicine, Washington, DC; Craven et al., 1999; Prybylowski et al., 2002). GFP-PSD-95 mutagenesis, the cloning of the C-terminus of the GluN2B subunit (amino acid 1086-1482) into pGEX-4T1 expression vector and that of the FLAG-tagged PSD-95 into pcDNA3.1 vector were performed by PCR amplification using Pfx DNA polymerase (Invitrogen) and the appropriate oligonucleotides. All mutations were fully sequenced to exclude the possibility of second site mutations. HA-tagged neuroligin 1 (NL1) in pCAG was kindly provided by Dr. Scheiffele (Biozentrum, University of Basel, Basel, Swizerland). pGEX4T1 plasmids containing Pin1WT, Pin1Y23A, Pin1C113A, and Pin1 $\Delta$ PPIase and FLAG-tagged Pin1WT and Pin1Y23A versions were previously described (Zacchi et al., 2002; Zita et al., 2007). To produce a high yield of in vitro transcription/ translated His-tagged PSD-95 the cDNA of PSD-95 was cloned into pT7CFE1-CHis plasmid which is optimized to use with the 1-Step Human In Vitro Protein Expression System (ThermoFisher Scientific).
Cell culture. HEK293T cells were cultured at $37^{\circ} \mathrm{C}$ under a $5 \% \mathrm{CO}_{2}$ atmosphere in DMEM supplemented with $10 \%$ fetal bovine serum. They were transiently transfected with various plasmids using polyethylenimine linear (Polysciences) and collected 24-48 h after transfection. Pin $1^{+/+}$and Pin $1^{-1-}$ primary hippocampal neurons were prepared as previously described (Antonelli et al., 2014) from either sex. For transfection experiments mouse hippocampal neurons were transiently transfected with the indicated plasmids using Effectene (Qiagen) according to the manufacturer's protocol.

PiB treatment. To inhibit Pin 1 catalytic activity, the chemical inhibitor PiB (diethyl-1,3,6,8-tetrahydro-1,3,6,8-tetraoxobenzol-phenanthroline-2,7diacetate) was added to cultured hippocampal neurons or it was stereotaxically injected in the hippocampus, and left to act for $48 \mathrm{~h}$ at a concentration of $2.5 \mu \mathrm{M}$. PiB was purchased from Calbiochem and resuspended in DMSO.

GST pull-down, coimmunoprecipitation, Western blot analysis, and synaptic protein extraction. GST pull-down and MPM-2-mediated immunoprecipitation were performed as previously described (Zacchi et al., 2002; Zita et al., 2007). Calf intestine phosphatase (CIP; 20 U/ml, New England Biolabs) was added to protein extracts for $1 \mathrm{~h}$ at $30^{\circ} \mathrm{C}$. For Pin1-FLAG (wt and Y23A) and GFP-PSD-95 coimmunoprecipitation, transfected cells were lysed in a buffer containing $50 \mathrm{~mm}$ Tris- $\mathrm{HCl}, \mathrm{pH} 7.5,100 \mathrm{~mm} \mathrm{NaCl}$, $0.1 \%$ Tween 20, 10\% glycerol, $10 \mathrm{~mm}$ EDTA, $2 \mathrm{~mm} \mathrm{MgCl}_{2}$, and protease inhibitor mixture. Extracts were incubated for $2 \mathrm{~h}$ at $4^{\circ} \mathrm{C}$ with the antiFLAG antibody (Sigma-Aldrich, clone M2) and immunocomplexes captured using Protein G Sepharose 4 fast Flow (GE Healthcare). NL1-HA and GFP-PSD-95 transfected cells were lysate in buffer CHAPS containing $50 \mathrm{~mm}$ Tris- $\mathrm{HCl}, \mathrm{pH}$ 7.5, 1 mм EDTA, $150 \mathrm{~mm} \mathrm{NaCl}$, 0.5\% CHAPS, $10 \%$ glycerol, and protease inhibitor mixture, and then immunoprecipitated with anti-HA affinity resin (Pierce). Coimmunoprecipitation of native PSD-95/Pin1 and PSD-95/NL1 complexes were obtained from p15 Pin $1^{+/+}$and Pin $1^{-/-}$brain homogenates (from either sex) in buffer CHAPS (as above), whereas PSD-95/NMDAR complexes were isolated using a chemical crosslinking approach (Poulopoulos et al., 2009). In all experiments, native complexes were immunoprecipitated using the antiPSD95 (Abcam). The following antibodies were used in Western blot analysis: rabbit anti-GluN1 (Sigma-Aldrich), rabbit anti-GluN2B (Alomone Labs), monoclonal anti-PSD95 (Abcam and NeuroMab), monoclonal anti-FLAG M2 (Sigma-Aldrich), and monoclonal anti-GFP (NeuroMab). Western blot image acquisition was performed using the ECL detection kit and the Alliance 4.7 software (UVITECH). Quantifications were performed using the UVI band imager software (GE Healthcare) as described previously (Antonelli et al., 2014). PSD enriched extracts were prepared by using the Syn-PER Extraction Reagent (ThermoFisher Scientific) as previously described (Antonelli et al., 2014). $p$ values were calculated using the Student's $t$ test with a two-tailed distribution on samples.

Subtilisin proteolysis. In vitro transcription/translated His-PSD-95 (IVT His-PSD-95) was purified on TALON Metal affinity resin (ThermoFisher Scientific) and phosphorylated upon $1 \mathrm{~h}$ incubation with a cell lysate derived from Pin $1^{-1-}$ mouse embryo fibroblasts. Equal amount of phosphorylated IVT His-PSD-95 were then incubated with $100 \mathrm{ng}$ of either GST, GST-Pin1, GST-Pin1-C113A, and GST-Pin1- $\Delta$ PPIase in a buffer containing $50 \mathrm{~mm}$ HEPES, pH 7.5, $100 \mathrm{~mm} \mathrm{NaCl}, 1 \mathrm{~mm} \mathrm{MgCl}_{2}$, supplemented with $100 \mu \mathrm{M}$ ATP and phosphatase inhibitors. After 30 min incubation at room temperature, reaction mixtures were cooled on ice, and subtilisin $(6.0 \mathrm{ng} / \mu \mathrm{l}$, Sigma-Aldrich) was added for a further 5 $\min$ at $4^{\circ} \mathrm{C}$. The reaction was stopped by the addition of boiling sample buffer, and the proteolytic fragments were resolved by $10 \%$ SDS-PAGE and visualized by Western blot analysis.

Immunocytochemistry, confocal microscopy, and image analysis. Hippocampal neurons grown on glass coverslips were fixed at 15 DIV with cold methanol for $5 \mathrm{~min}$, blocked by incubation with $10 \%$ normal goat serum in PBS. Antibodies were diluted in 5\% normal goat serum/PBS. Secondary antibodies included anti-isotypic fluorophore-conjugated antibodies AlexaFluor 488, AlexaFluor 594 and streptavidin-AlexaFluor 405 at dilutions of 1:1000 (Invitrogen). The following commercially available antibodies were used in immunocytochemistry: mouse antiPSD95 (Sigma-Aldrich), guinea pig anti-vGLUT1 (Millipore Bioscience Research Reagents), rabbit anti-GluN1 (Sigma-Aldrich). Fluorescence 
images were acquired on a TCS-SP confocal laser-scanning microscope (Leica) with a $40 \times 1.4$ NA oil-immersion objective, and additionally magnified fivefold with the pinhole set at 1 Airy unit. All the parameters used in confocal microscopy were consistent in each experiment, including the laser excitation power, detector and offset gains, and the pinhole diameter. Quantification of immunofluorescence data were performed using the Volocity3D Image Analysis Software (PerkinElmer; Antonelli et al., 2014).

Hippocampal slice preparation. All experiments were performed in accordance with the European Community Council Directive of November 24, 1986 (86/609EEC) and approved by the local authority veterinary service and by International School for Advanced Studies ethical committee.

Transverse hippocampal slices (300 $\mu \mathrm{m}$ thick) were obtained from postnatal day (P)10-P15 mice (male and female) using a standard protocol (Gasparini et al., 2000). Briefly, after being anesthetized with $\mathrm{CO}_{2}$, animals were decapitated. The brain was quickly removed from the skull and placed in ice-cold artificial CSF (ACSF) containing the following (in mM): $130 \mathrm{NaCl}, 25$ glucose, $3.5 \mathrm{KCl}, 1.2 \mathrm{NaH}_{2} \mathrm{PO}_{4}, 25 \mathrm{NaHCO}_{3}, 2 \mathrm{CaCl}_{2}$, and $1.3 \mathrm{MgCl}_{2}$, saturated with $95 \% \mathrm{O}_{2}$ and $5 \% \mathrm{CO}_{2}$ (pH 7.3-7.4). Transverse hippocampal slices were cut with a vibratome and stored at room temperature $\left(22-24^{\circ} \mathrm{C}\right)$ in a holding bath containing the same solution as above. After incubation for at least $45 \mathrm{~min}$, an individual slice was transferred to a submerged recording chamber and continuously superfused with oxygenated ACSF at a rate of 3-4 $\mathrm{ml} / \mathrm{min}^{-1}$.

Electrophysiological recordings and data analysis. Whole-cell patchclamp recordings (in voltage-clamp configuration) were performed from CA1 pyramidal cells, visualized with an upright microscope, using a patch-clamp amplifier (Axopatch 1D amplifier, Molecular Devices). The stability of the patch was checked by repetitively monitoring the input and series resistance during the experiment. Cells exhibiting $>20 \%$ changes in series resistance were excluded from the analysis.

AMPAR- and NMDAR-mediated EPSCs, evoked in CA1 principal cells by stimulation of Schaffer collateral $(100 \mu$ s duration pulses at 0.1 $\mathrm{Hz})$ were recorded at room temperature $\left(20-22^{\circ} \mathrm{C}\right)$ from a holding potential of -60 and $+40 \mathrm{mV}$, respectively, in the presence of bicuculline methiodide $(10 \mu \mathrm{M})$ to block $\mathrm{GABA}_{\mathrm{A}} \mathrm{R}$-mediated IPSCs. In these experiments, stimulation was set at such intensity to produce half-maximal responses. To avoid contamination with possible epileptiform discharges originating from the $\mathrm{CA} 3$ region, slices were routinely cut between the CA3 and the CA1 region. The NMDA-AMPA ratio was analyzed in two steps. First, stable synaptic responses were obtained at $-60 \mathrm{mV}$ (the amplitude of these responses was the AMPAR-specific component). Next, the holding potential was changed to $+40 \mathrm{mV}$ and dual component EPSCs were collected. At $50 \mathrm{~ms}$ poststimulus, when the AMPAR contribution was negligible, the amplitude of the dual component EPSC was interpreted as the NMDAR-specific component (Etherton et al., 2011).

In cultured hippocampal cells, AMPAR-mediated EPSCs were evoked at $-60 \mathrm{mV}$ by square pulses ( $5 \mathrm{~ms}$ duration) delivered at $0.1 \mathrm{~Hz}$ through a glass electrode filled with extracellular solution positioned in the vicinity of the neuron to be stimulated. NMDAR-mediated EPSCs were recorded at $+40 \mathrm{mV}$ after blocking the AMPA-mediated components with 6,7-dinitroquinoxaline-2,3-dione (DNQX; $10 \mu \mathrm{M}$ ). At the end of the experiment, 2-amino-5-phosphonopentanoic acid (AP-5) was added to confirm that, in these conditions, the recorded current was mediated by NMDARs.

Drugs used were as follows: DNQX, bicuculline methiodide, AP-5, and threo-ifenprodil-hemitartrate, all purchased from Tocris Bioscience. The ifenprodil experiments were done on slices maintained in a $\mathrm{Mg}^{2+}$ free solution containing both bicuculline and DNQX $(20 \mu \mathrm{M})$, to block $\mathrm{GABA}_{\mathrm{A}}$ and AMPA receptors, respectively. Ifenprodil was added only if cell responses were stable for at least $5 \mathrm{~min}$.

Data were acquired at $20 \mathrm{kHz}$, filtered with a cutoff frequency of $3 \mathrm{kHz}$ and analyzed off-line using Clampfit 10.0 (Molecular Devices). Amplitude, kinetics, and area of EPSCs were measured on averaged traces (usually 10 consecutive EPSCs). Only single-peaked, fast-latency $(<2$ $\mathrm{ms}$ ) events were included to ensure monosynaptic responses. The decay of AMPA-mediated EPSCs was fitted with a single exponential function:

$$
I(t)=A \exp (-t / \tau)
$$

where $I(t)$ is the current as a function of time, $A$ is the amplitude at time 0 , and $\tau$ is the time constant.

The decay of NMDA-mediated EPSCs was fitted with two exponential functions:

$$
I(t)=A_{\text {fast }} \exp \left(-t / t_{\text {fast }}\right)+A_{\text {slow }} \exp \left(-t / t_{\text {slow }}\right),
$$

where $A_{\text {fast }}$ and $A_{\text {slow }}$ are the fraction of the fast and slow component, respectively; $\tau_{\text {fast }}$ and $\tau_{\text {slow }}$ are the fast and the slow time constants. Data are presented as mean \pm SEM. Statistical comparison was performed using the unpaired Student's $t$ test. A $p$ value $<0.05$ was considered as statistically significant.

Golgi staining, intrahippocampal injection of PiB, and spine morphology assessment. For Golgi staining five $\mathrm{Pin}^{-/-}$and $\mathrm{Pin}^{+/+}$littermates were intracardially perfused with $0.9 \%$ saline solution. Brains were rapidly collected and immersed in Golgi solution (1\% potassium dichromate, $1 \%$ mercuric chloride, and $0.8 \%$ potassium chromate in distilled water) for $5 \mathrm{~d}$ at room temperature. After a rapid $(24 \mathrm{~h})$ passage in $30 \%$ sucrose solution, $100 \mu \mathrm{m}$ coronal sections were cut through a vibratome and then mounted on gelatin slides for staining according to the Gibb and Kolb method (Gibb and Kolb, 1998). Spine analysis was performed on apical dendrites of neurons lying on the CA1 region in the ventral hippocampus using the public domain ImageJ software (NIH) according to previously described protocols (Middei et al., 2012). For intrahippocampal injection of $\mathrm{PiB}$ (dissolved in DMSO) or vehicle (DMSO alone) three adults (2 months old) Thy-1-GFP mice (The Jackson Laboratory) were stereotaxically injected with $\mathrm{PiB}(2.5 \mu \mathrm{M}$ in DMSO $0.1 \%)$ in one hippocampus and with vehicle (DMSO $0.1 \%$ ) in the opposite one (stereotaxic coordinates: $\mathrm{AP}=-2, \mathrm{~L}=+1.7, \mathrm{DV}=-1.7$ ). Forty-eight hours after injection mice were intracardially perfused with $4 \%$ PFA. Brains were removed from the skulls and cryoprotected overnight in $30 \%$ sucrose. Forty-micrometer-thick sections were collected and mounted for confocal imaging. GFP-positive neurons surrounding the site of PiB or DMSO injection were identified along the CA1 region of the hippocampus following the track of the injection cannula. For each neuron, five nonoverlapping dendritic segments (average length $20 \mu \mathrm{m}$ ) were randomly selected for image acquisition at $63 \times$ /zoom $1 \times$ magnification using a confocal microscope (Zeiss LSM700). IMARIS Software (v7.6.5) was used to automatically count the number of spines along dendritic segments.

\section{Results \\ PSD-95 recruits Pin1 at consensus sites located between PDZ2 and PDZ3 domains}

Proteins phosphorylated on serine/threonine-proline motifs represent potential targets for the peptidyl-prolyl isomerase cis-/ trans isomerize Pin1 (Lu and Zhou, 2007). To examine whether PSD-95 undergoes proline-directed phosphorylation we took advantage of the mitotic phosphoprotein monoclonal-2 (MPM-2) antibody which specifically recognizes phosphorylation events generating Pin 1 consensus epitopes in several targets (Davis et al., 1983). In immunoprecipitation experiments MPM-2 antibody was able to precipitate endogenous PSD-95 from mouse brain (Fig. 1A, left), as well as FLAG-PSD-95 expressed in HEK293T cells (Fig. $1 A$, right).

The observation that PSD-95 undergoes proline-directed phosphorylation prompted us to ascertain whether it interacts with Pin 1 . To this aim, we initially performed coimmunoprecipitation experiments on ectopically expressed GFP-PSD95 and Pin1-FLAG. Under these conditions we observed that PSD-95 is selectively pulled-down by Pin1-FLAG and not by control mouse IgG, thus indicating that it interacts with Pin1 (Fig $1 B$, left). In addition, native PSD-95/Pin 1 complexes were detected in mouse brain homogenates after immunoprecipitation of endogenous PSD-95 derived from Pin $1^{+/+}$mice, but not in control precipi- 
A

B

Pin1+/+ Pin1-/-

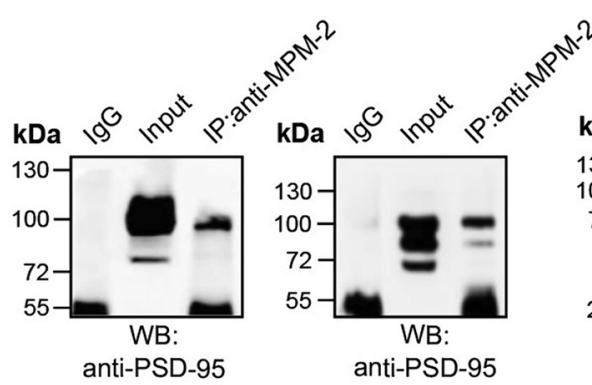

C
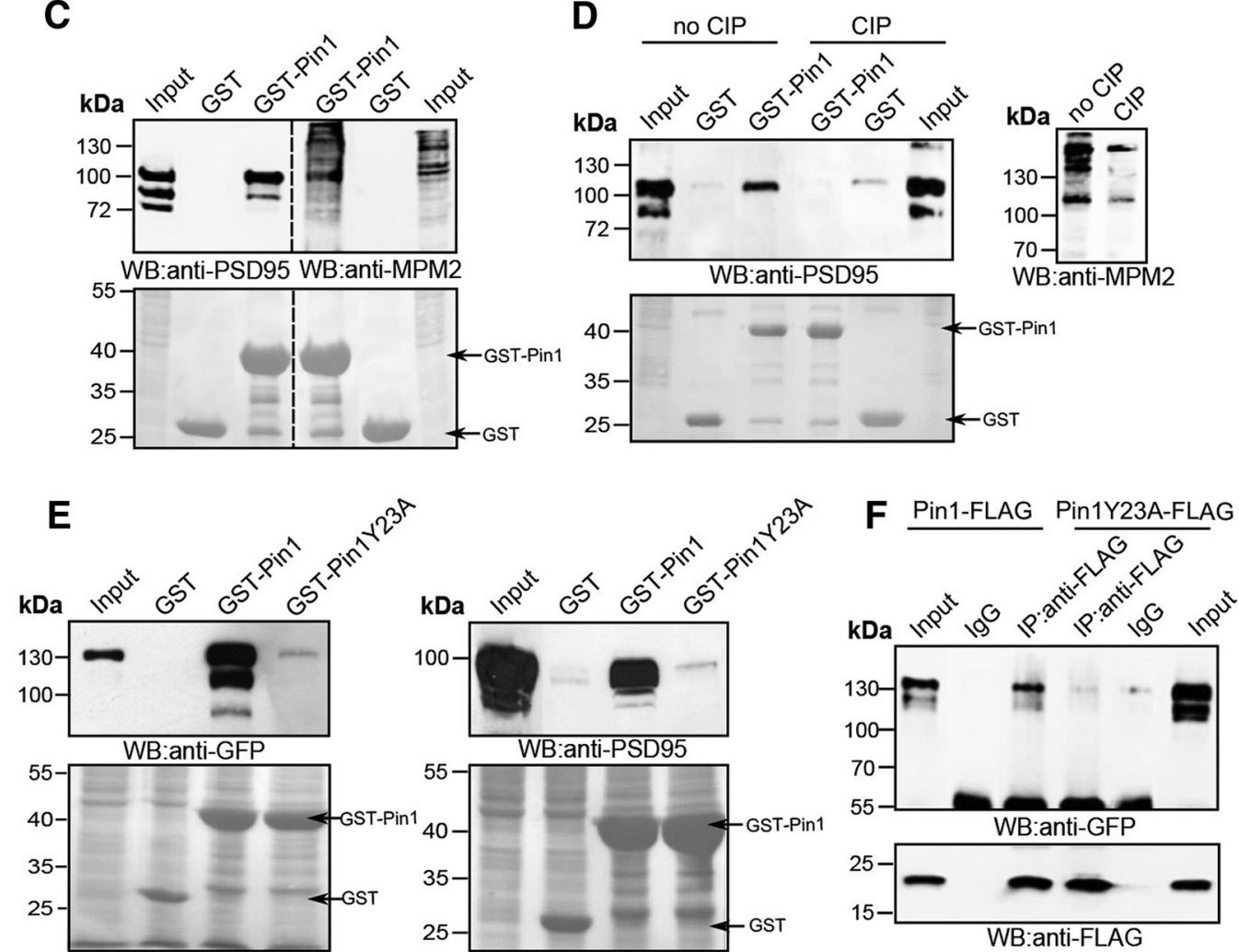

Figure 1. Pin1 interacts with PSD-95. A, Representative immunoprecipitation (IP) of endogenous PSD-95 from mouse brain (left) or FLAG-tagged PSD-95 ectopically expressed in HEK293Tcells (right) using the anti-MPM-2 antibody $(n=4)$. Nitrocellulose membranes were probed with anti-PSD-95 antibody. $\boldsymbol{B}$, Lysates of HEK293T cells transfected with Pin1-FLAG and GFP-PSD-95WT were immunoprecipitated with anti-FLAG antibody. Immunoprecipitates were analyzed by Western blotting using anti-FLAG and anti-PSD-95 monoclonal antibodies (left; $n=4$ ). Coimmunoprecipitation of endogenous PSD-95/Pin 1 complexes from brain homogenates of Pin $1^{+/+}$or Pin $1^{-1-}$ mice was performed using anti-PSD-95 antibody. Western blots were performed with anti-PSD-95 and anti-Pin1 antibodies (right; $n=3$ ). C, Extracts of HEK293T cells transfected with FLAG-PSD-95 were incubated with GST and GST-Pin1. Independent experiments were run in parallel to perform immunoblots using anti-PSD-95 and anti-MPM2 antibodies $(n=5)$. $\boldsymbol{D}$, Similar pull-down were performed as described in $C$ but one-half of the lysate was treated with CIP before incubation with

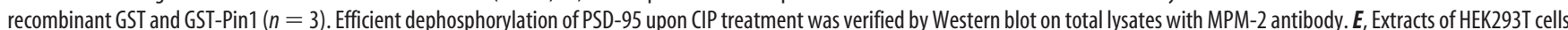
transfected with GFP-PSD-95WT (left) or mouse brain homogenates (right) were incubated with GST, GST-Pin1, and GST-Pin1Y23A and immunoblotted with anti-PSD-95 and anti-GFP antibodies, respectively $(n=4)$. $F$, Lysate of HEK293T cells cotransfected with GFP-PSD-95 together with Pin1-FLAG or Pin1Y23A-FLAG were immunoprecipitated with anti-FLAG antibody. Immunoprecipitates were analyzed by Western blotting using anti-FLAG and anti-GFP monoclonal antibodies $(n=4)$. In each pull-down, GST was used as negative control and the bottom represents the levels of GST and GST-Pin1 used in the assays (Ponceau staining). In each immunoprecipitation experiment Mouse lgG was used as negative control.

tates nor in the absence of Pin 1 expression (Fig. $1 B$, right). This interaction strictly depends on PSD-95 phosphorylation, being the MPM-2-immunoreactive band pulled down by GST-Pin1 corresponding to the apparent PSD-95 molecular weight (Fig. 1C). Moreover GST-Pin1 binding to PSD-95 was completely abolished upon CIP treatment (Fig. 1D). The phosphodependency of Pin1/PSD-95 complex formation was further demonstrated by the lack of binding between endogenous or ectopically expressed PSD-95 and GST-Pin1Y23A, a mutant dis- rupted in phospho-Ser/Thr-binding activity (Fig. 1E, left and right, respectively; Lu et al., 1999), results then confirmed in coimmunoprecipitation experiments (Fig. $1 F$ ).

PSD-95 contains six potential Pin1 recognition motifs organized in two clusters, one located on the N-terminal (T19/S25/ S35), whereas the other on the linker region connecting PDZ2 to PDZ3 domains (T287/S290/S295; Fig. 2A). To identify the Pin 1 binding sites on PSD-95, we initially generated PSD-95 deletion constructs lacking either the first cluster (PSD-95019-35), the 


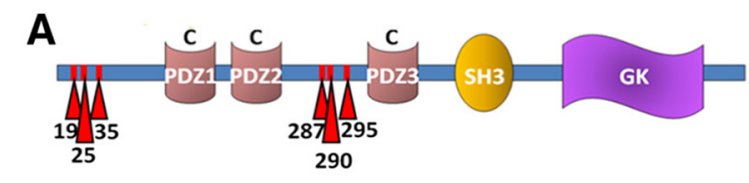

B GFP-PSD-95 GFP-PSD-95 GFP-PSD-95
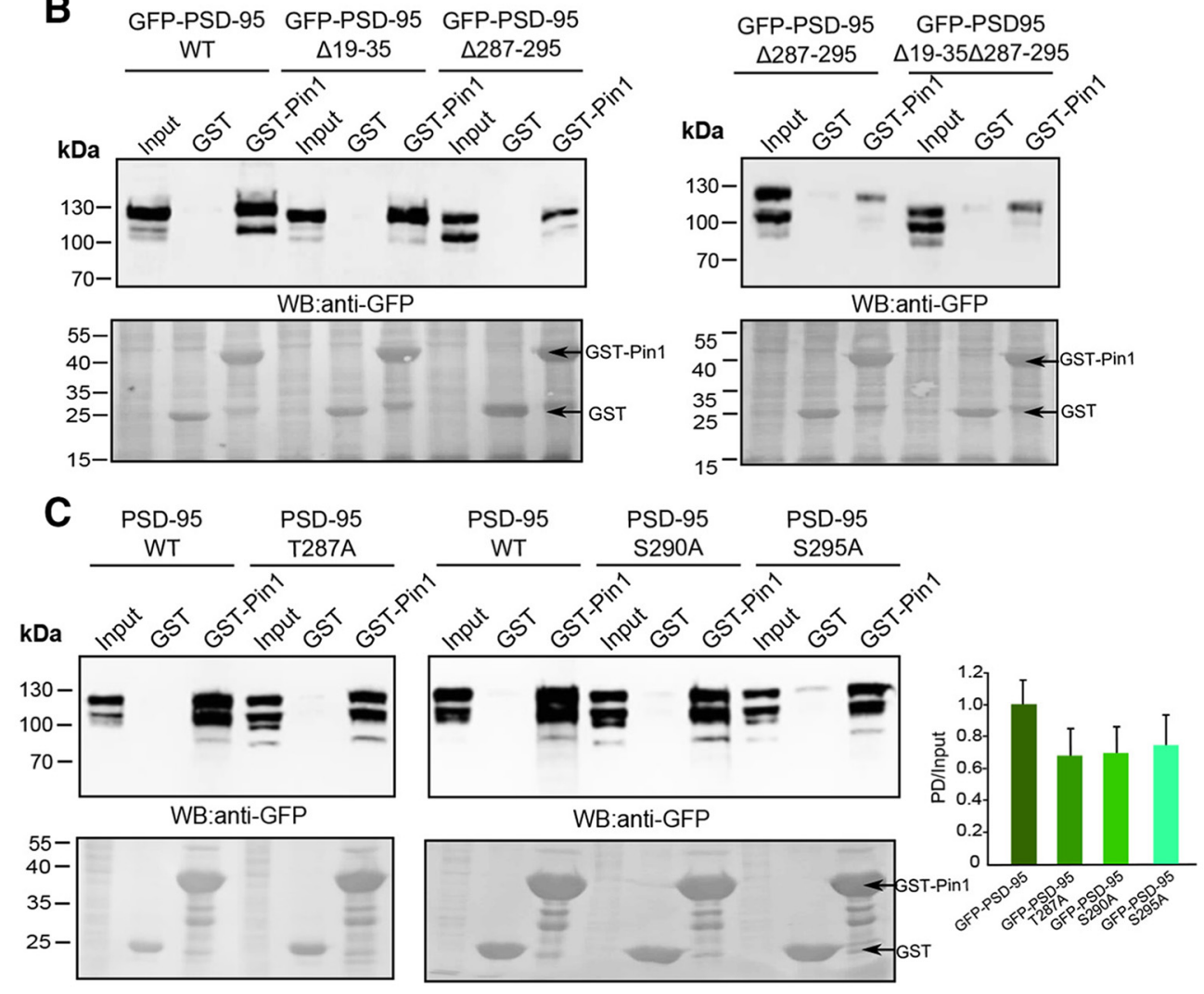

S290A

S295A

D
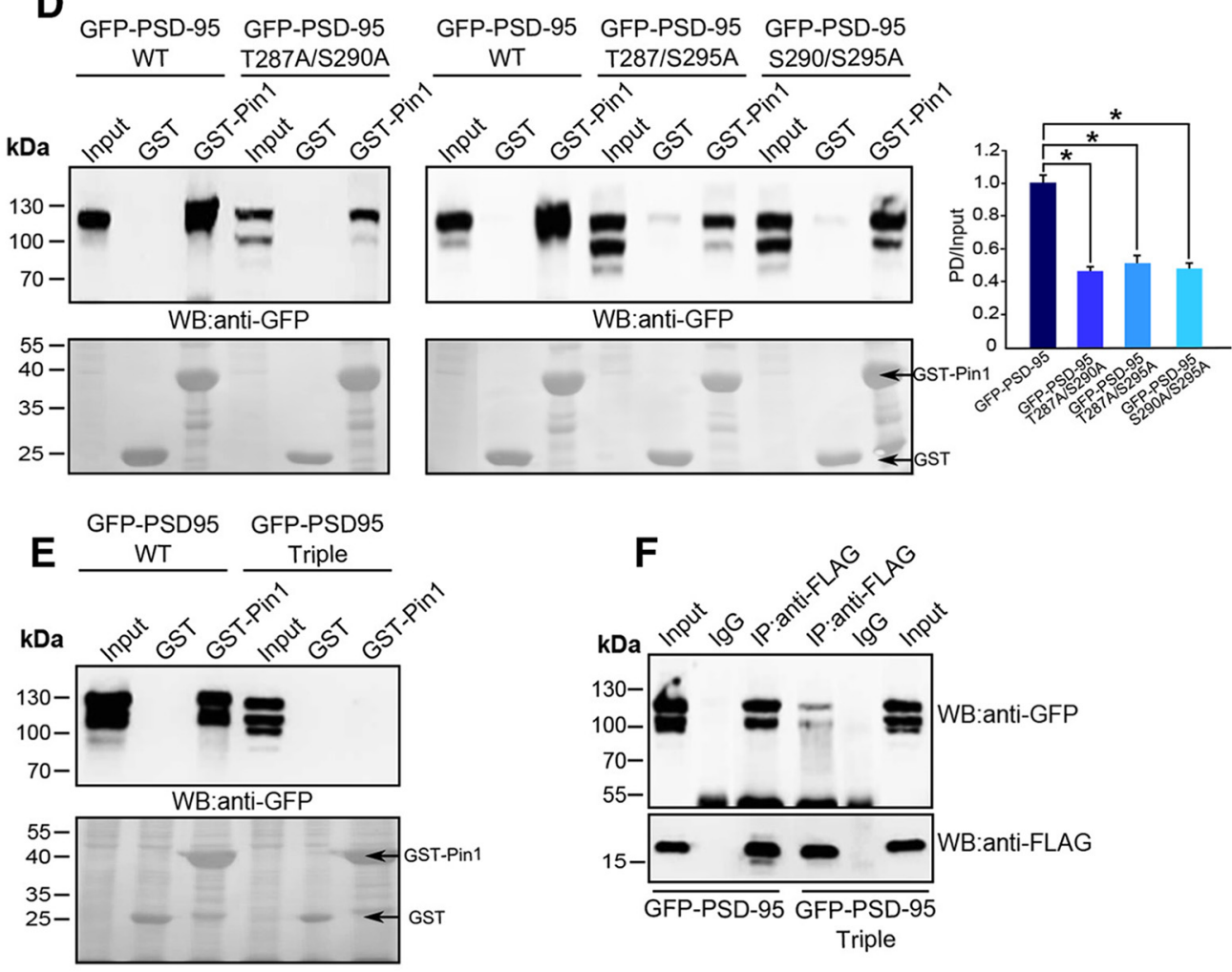
second (PSD-95 287-295), or both and tested them for interaction with GST-Pin1. PSD-95 19-35 was associated with Pin1 to the same extent as wild-type PSD-95, whereas PSD-95 $\Delta 287-295$ only poorly interacted with the chaperone molecule (Fig. $2 B$, left) and its binding capacity was not further dampened upon the additional removal of the $\mathrm{N}$-terminal sites (Fig. $2 B$, right).

To further validate the importance of this short stretch of amino acid residues (287-295) as Pin 1 recruitment domain, we performed a serine/threonine to alanine scan mutagenesis at each putative Pin 1 consensus site to generate singles (PSD-95T287A, PSD-95S290A, PSD-95S295A), doubles (PSD-95S287A/S290A), and the triple- (PSD-95S287A/S290A/S295A) mutants to be tested in the GST-Pin 1 pull-down assays. Under these conditions, whereas single-point mutants exhibited a similar affinity for Pin1 as the wild-type protein (Fig. 2C), double-mutants showed a significant reduction in binding (Fig. $2 D$ ), which was almost abolished upon alanine substitution at all three sites both in GST-Pin 1 pull-down (Fig. 2E) and in coimmunoprecipitation (Fig. 2F), thus suggesting that all of them contribute to Pin1 recruitment.

\section{Pin1 elicits structural rearrangements in PSD-95}

To verify whether the prolyl-isomerase activity of Pin 1 induces conformational changes in PSD-95 we performed a partial proteolysis assay on in vitro-translated PSD-95 (IVT His-PSD-95). This assay relies on the observation that Pin1-dependent structural changes confer substrate resistance to subtilisin serine endopeptidase proteolysis (Manganaro et al., 2010; Yoon et al., 2014). Because IVT His-PSD-95 resulted only poorly phosphorylated by the mammalian cell-free system used (data not shown), we first incubated His-PSD-95 with a cell lysate derived from Pin $1^{-1-}$ embryo fibroblasts to promote its proline-directed phosphorylation before Pin 1 addition and protease cleavage. Under these conditions His-PSD-95 become resistant to protease digestion only upon incubation with recombinant GST-Pin1wt, being the Pin 1 mutants deficient in prolyl-isomerase activity, but fully competent in substrate binding, completely ineffective as control GST (Fig. 3). These data altogether strongly indicate that Pin1 not only is recruited by PSD-95 in a phosphorylationdependent manner but it also promotes structural rearrangements that may impinge on its function at PSD.

\section{Pin 1 action on PSD-95 alters the ability of the scaffold molecule to interact with the NMDA receptors}

The close proximity of the identified Pin 1 recruitment motifs to PSD-95 PDZ domains known to be involved in GluN2A/B and NL1 interaction raises the possibility that a conformational shift in this region may affect the PDZ binding affinity for the corre-

\section{$\leftarrow$}

Figure 2. Pin1 interacts with PSD-95 at T287, S290, and S295 consensus motifs. $\boldsymbol{A}$, Schematic representation of PSD-95 domains (PDZs, brown; SH3, yellow; GK, purple). Putative Pin1 sites are indicated by red arrows. $\boldsymbol{B}$, Extracts of HEK293T cells transfected GFP-PSD-95WT or the indicated deletion mutants were incubated with GST or GST-Pin1 and immunoblotted with anti-GFP antibody. C-E, Extracts of HEK293T cells transfected with GFP-PSD-95WT and the indicated single-, double-, and triple-point mutants were incubated and processed as in $\boldsymbol{B}$. Histograms represent the amount of PSD-95 mutants pulled-down (PD) by equal amount of GST-fusion probe normalized to their expression level (Input). ( $n=4$; mean values \pm SD; $p>$ 0.05 for single-mutants, ${ }^{*} p<0.05$ for double-mutants, Student's $t$ test). $\boldsymbol{F}$, Extracts of HEK293T cells cotransfected with Pin1-FLAG and GFP-PSD-95 or PSD-95 triple-mutant were immunoprecipitated with anti-FLAG or mouse $\mathrm{Ig} G$ as negative control and immunoblotted with anti-GFP or anti-FLAG antibodies, respectively $(n=3)$. $\boldsymbol{B}-\boldsymbol{E}$, Bottom, Levels of GST fusion proteins used in the assays (Ponceau staining).

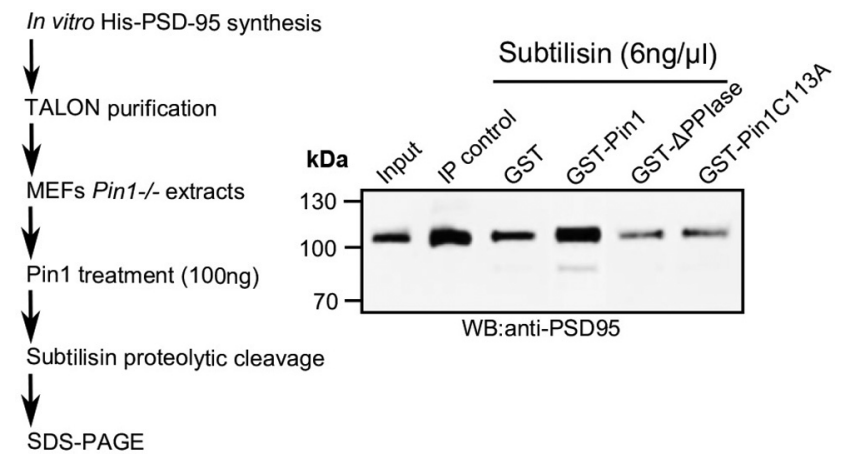

Figure 3. Pin1 catalyzes a conformational change in PSD-95. Left, Schematic outline of the experimental flow-chart. Right, Purified IVT His-PSD-95 was treated with a cell lysate of Pin $1^{-1-}$ MEFs to sustain proline-directed phosphorylation, followed by incubation with GST, GST-Pin1, GST-Pin1 $\triangle$ PPase, or Pin1C113A. Upon subtilisin addition the residual IVT His-PSD-95 was visualized by SDS-PAGE followed by Western blot analysis using a PSD-95 antibody $(n=5)$.

sponding binders. The first two PDZ domains are known to directly interact with the C-terminal of the GluN2A and/or GluN2B subunits of NMDARs (Kornau et al., 1995; Niethammer et al., 1996), whereas PDZ3 with the cell-adhesion molecule NL1 (Irie et al., 1997). Neither detectable differences in GFP-PSD95wt and PSD-95 triple-mutant precipitated by NL1-HA were found (Fig. 4A) nor in endogenous NL1 pulled down by PSD-95 in the presence or absence of Pin 1 expression (Fig. 4B), indicating that PDZ3 was not involved. By contrast, PSD-95 triple-mutant showed an enhanced association with the GluN2B compared with PSD-95wt (Fig. 4C) in GST-based pull-down assays (only the C-terminus of GluN2B). This observation prompted us to investigate how endogenous PSD-95 complexes with NMDARs in the presence or in the absence of Pin 1. As shown in Figure 4D, in Pin $1^{-1-}$ hippocampal extracts, the amount of GluN2B receptor subunit coprecipitated by PSD-95 was increased by $20.9 \pm$ 2.02\% compared with Pin1-expressing neurons (Fig. 4D).

To test whether the enhanced PSD-95/GluN2B complex formation occurs at synaptic sites, we analyzed the expression levels of PSD-95, GluN1 (the obligatory subunit of all NMDARs; Paoletti et al., 2013), and GluN2B in synaptosomal fractions isolated from Pin $1^{+/+}$and Pin $1^{-/-}$hippocampus. As shown in Figure $4 E$, quantitative immunoblot analysis unveiled that the amount of GluN1 and GluN2B subunits was significant increased in Pin $1^{-1-}$ mice, being the synaptic enrichment (synaptic fraction vs homogenate) for GluN1 of $18 \pm 3 \%$ and GluN2B of $23 \pm 4 \%$. Altogether, these data indicate that Pin 1 negatively modulates the synaptic enrichment of NMDARs via PSD-95 prolyl-isomerization.

\section{Pin 1 affects NMDA-mediated synaptic signaling}

To investigate the functional consequences of Pin1-dependent regulation of NMDARs signaling at excitatory synapses, the NMDA-AMPA ratio of synaptic currents evoked in CA1 principal cells by Schaffer collateral stimulation was analyzed in both genotypes. In comparison with control littermates, Pin $1^{-1-}$ mice exhibited larger NMDA-mediated synaptic responses (Fig. 5A). On average, the peak amplitudes of AMPAR-mediated EPSCs were similar in both genotypes $(103 \pm 10 \mathrm{pA}$ and $107 \pm 12 \mathrm{pA}$ in Pin $^{+/+}, n=17$ and Pin $1^{-/-}$mice, $n=16$, respectively; $p=0.8$ ), whereas NMDA-mediated EPSCs were enhanced in Pin $1^{-1-}$ mice $\left(48 \pm 5 \mathrm{pA}\right.$ and $71 \pm 13 \mathrm{pA}$ in Pin $1^{+/+}$and Pin $1^{-/-}$mice, respectively; $\left.{ }^{\star} p<0.05\right)$. As expected, the NMDA-AMPA ratio was significantly increased in Pin $1^{-1-}$ mice respect to controls 
A
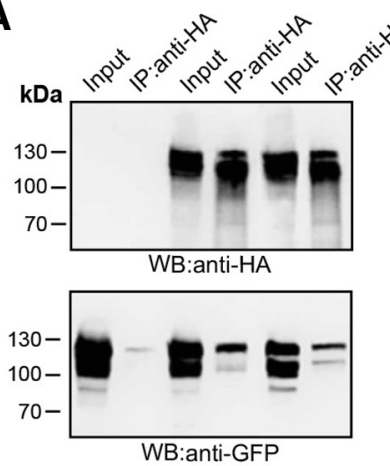

GFP-PSD-95 + + + + - -

$\mathrm{NL} 1-\mathrm{HA}--++++$

GFP-PSD-95 - - -++ Triple
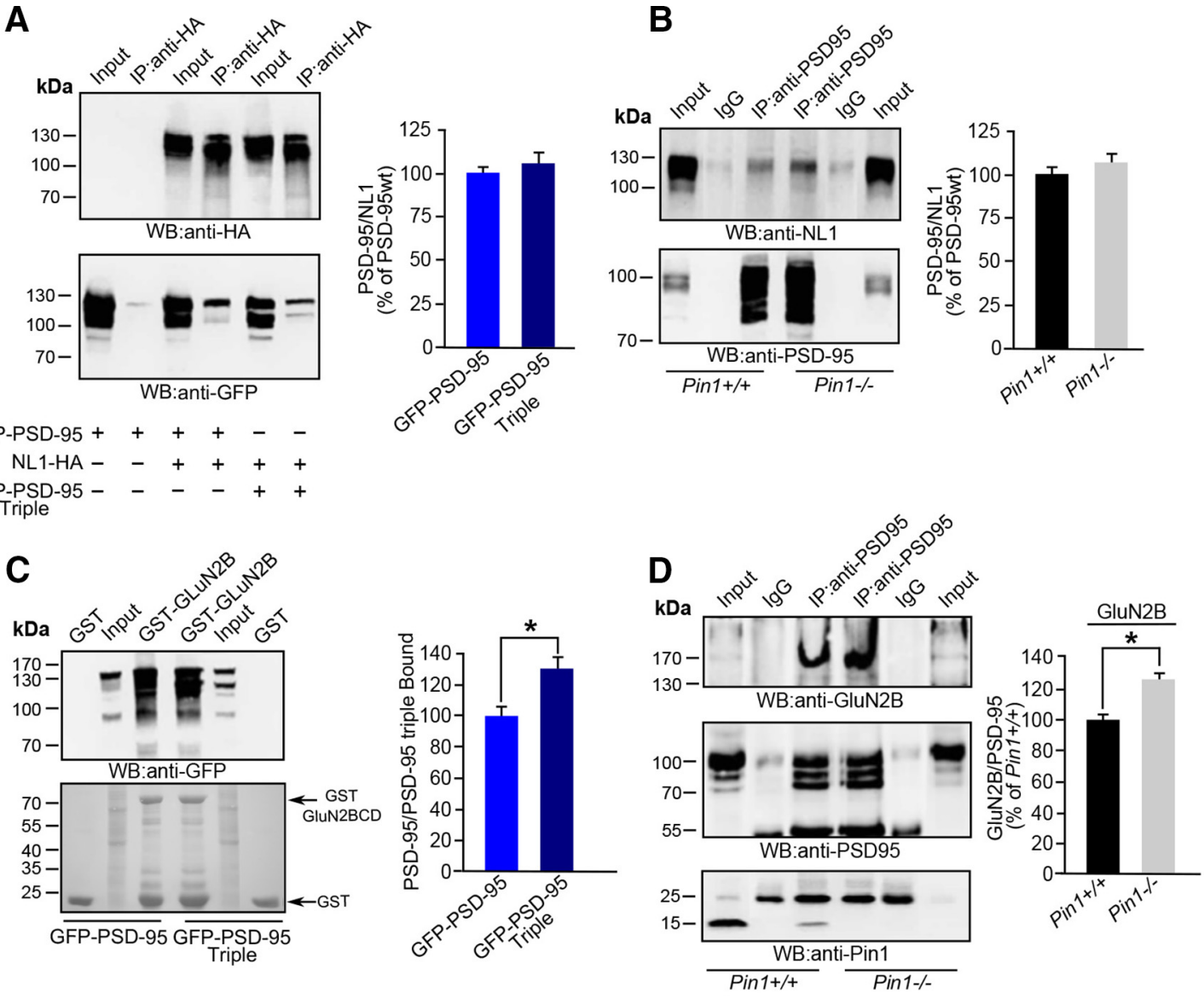

$\mathbf{E}$
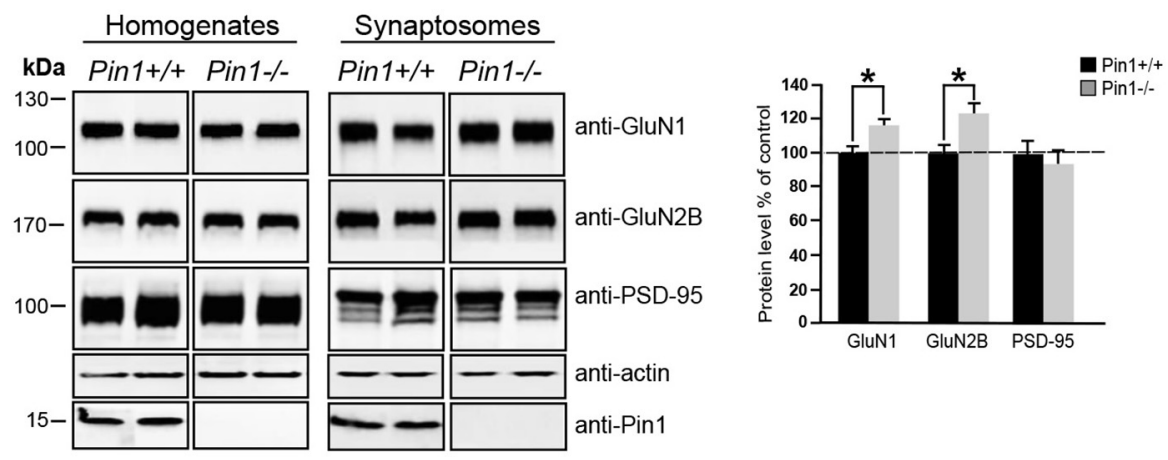

Figure 4. Pin1 modulates PSD-95's ability to complex with NMDARs at synapse. A, Extracts of HEK293T cells transfected with the indicated plasmids were immunoprecipitated with anti-HA antibody and immunoblotted with anti-GFP and anti-HA. Histogram shows the relative amount of PSD-95 or PSD-95 Triple mutant in complex with NL1-HA obtained from densitometric analysis $\left(n=4\right.$; mean values \pm SD, $p>0.05$, Student's $t$ test). B, Brain extracts from Pin $1^{+/+}$and Pin $1^{-/-}$were immunoprecipitated with anti-PSD-95 antibody and immunoblotted with anti-PSD-95 and anti-NL1. Histogram shows the relative amount of NL1 in complex with PSD-95 obtained as in $\boldsymbol{A}(n=4$, mean values \pm SD, $p>0.05$, Student's $t$ test). C, Extracts of HEK293T cells transfected with GFP-PSD-95 were incubated with GST and GST-GluN2B and immunoblotted with anti-GFP antibody. Bottom, Levels of GST fusion proteins (Ponceau staining). Histogram shows the amount of PSD-95 or PSD-95 Triple pulled-down as percentage of PSD-95WT $\left(n=5\right.$; mean values \pm SD, ${ }^{*} p<0.05$, Student's $t$ test). $\boldsymbol{D}$, Brain extracts from Pin $1^{+/+}$and Pin $1^{-1-}$ were immunoprecipitated with anti-PSD-95 antibody and immunoblotted with anti-GluN2B, anti-PSD-95 and anti-Pin1. Histogram shows the amount of GluN2B in complex with PSD-95 quantify as in C $(n=5$; mean values $\pm S D,{ }^{*} p<0.05$, Student's $t$ test). $\boldsymbol{E}$, Representative immunoblots of the indicated antigens extracted from the hippocampus of Pin $1^{+/+}$and Pin $1^{-1-}$ mice (littermates) in two different sets of experiments. On the right quantification of the indicated antigens. Actin represents loading control. ( $n=6$ littermate pairs, mean values $\pm S D,{ }^{*} p<0.05$, Student's $t$ test).

$\left(0.49 \pm 0.04\right.$ and $0.66 \pm 0.07$ in Pin $^{+/+}$and Pin $1^{-/-}$mice, respectively, ${ }^{*} p<0.05$; Fig. $5 B$ ), as well as the charges transfer through synaptic NMDARs (Fig. 5C), thus suggesting modifications in amplitude and/or shape.

NMDARs are assembled as heteromers that differ in subunits composition, giving rise to NMDARs with different biophysical and functional properties (Paoletti et al., 2013). In particular, NMDARs containing GluN2B-D subunits, exhibit slower deactivation kinetics respect to those containing GluN2A (Vicini et al.,
1998). Therefore, to investigate whether Pin 1 may alter the subunits composition of NMDARs, we measured the deactivation kinetics of NMDA- and AMPA-mediated synaptic currents in both genotypes. Although the decay of AMPA-mediated EPSCs could be fitted by a single exponential $\left(\tau_{\text {fast }}: 11 \pm 1\right.$ and $13 \pm 1 \mathrm{~ms}$ in Pin $1^{+/+}, n=16$ and Pin $1^{-1-}$ mice, $n=11$, respectively, $p=$ $0.21)$, that of NMDAR-mediated currents by two exponentials $\left(\tau_{\text {fast }}: 55 \pm 4\right.$ and $42 \pm 6 \mathrm{~ms}$ in Pin ${ }^{+/+}, n=16$ and Pin $1^{-1-}$ mice, $n=13$, respectively; $\tau_{\text {slow: }} 243 \pm 27$ and $218 \pm 32$ 
A

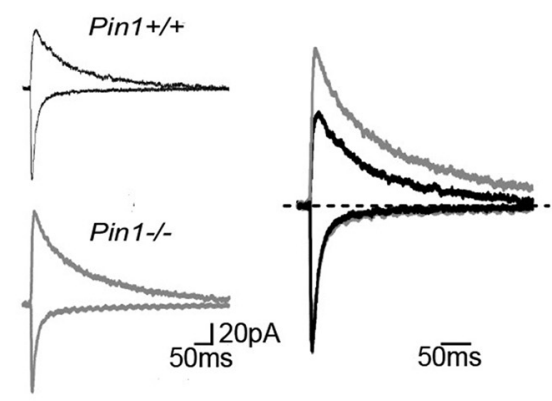

D

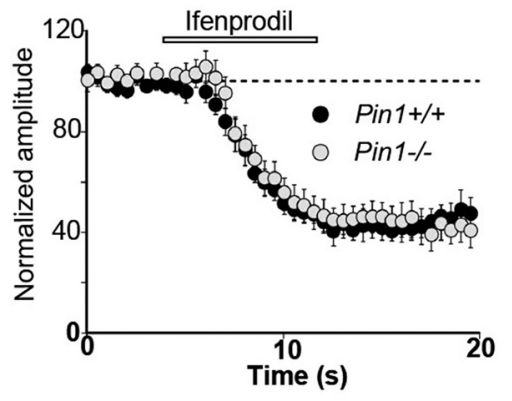

B

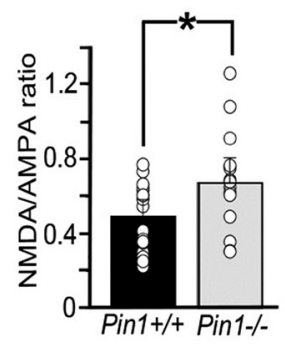

E

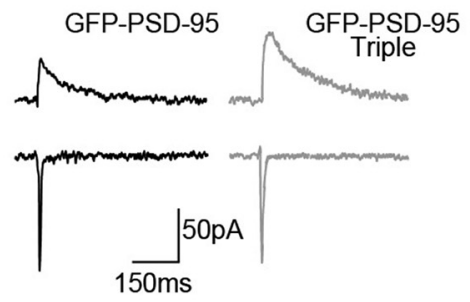

C

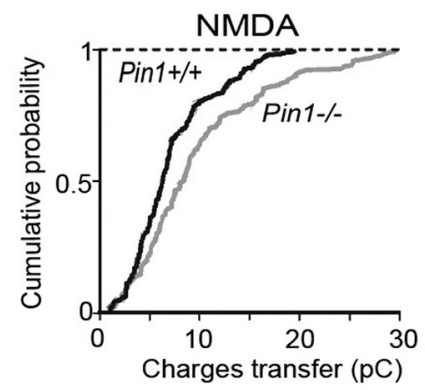

$\mathbf{F}$

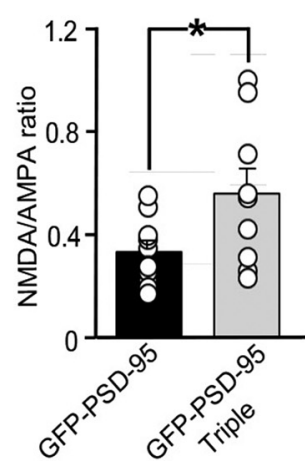

Figure 5. Pin1 controls synaptic signaling via NMDA receptors and regulates spine number and size. $A$, Sample traces of NMDAR- and AMPAR-mediated EPSCS recorded from CA1 principal cells in hippocampal slices of Pin $1^{+/+}$and Pin $1^{-/-}$at holding potentials of -60 and $+40 \mathrm{mV}$, respectively. Each trace is the average of 10 responses. On the right, the traces normalized to those mediated by AMPAR are superimposed. $\boldsymbol{B}$, Summary graphs of the NMDA/AMPA-mediated receptor response ratios. Data represent mean \pm SEM. 0 pen symbols are individual values. ${ }^{*} p<0.05$, Student's $t$ test. C, Cumulative probability plots of charge transfers through NMDAR-mediated currents $\left(^{*} p=0.003\right.$; Kolmogorov-Smirnov test). $\boldsymbol{D}$, Time course of ifenprodil action (open bar) on NMDAR-mediated synaptic currents from Pin $1^{+/+}(n=12)$ and Pin $1^{-1-}$ mice $(n=10)$. Each point represents the mean \pm SEM. $\boldsymbol{E}$, Examples of evoked AMPA- and NMDA-mediated EPSCs in cultured hippocampal cells from GFP-PSD-95wt (black) and GFP-PSD-95 triple-mutant (gray) transfected cells. F, Summary graphs of the NMDA-AMPA ratios of transfected neurons. Open symbols are individual values. ${ }^{*} p<0.05$, Student's $t$ test.

ms in Pin $1^{+/+}$and Pin $1^{-/-}$mice, respectively). Despite a certain degree of variability between the two genotypes, no significant differences in the decay time constants were observed $(p=0.1$ and $p=0.8$ for $\tau_{\text {fast }}$ and $\tau_{\text {slow }}$, respectively), suggesting that Pin 1 does not affect the composition of synaptic NMDAR subtypes. Moreover, treatment with ifenprodil, a selective antagonist of GluN2B-containing receptors (Williams, 1993), produced a similar depression of NMDA-mediated synaptic currents in both genotypes (Fig. 5D), further indicating that Pin 1 does not alter the subunits composition of NMDA receptors but it exerts its control on their total number. It is interesting to note that the NMDA-AMPA ratio was also increased in cultured hippocampal neurons transfected with GFP-PSD-95 triple-mutant compared with GFP-PSD-95wt $(0.56 \pm 0.09$ and $0.33 \pm 0.04$, respectively, ${ }^{\star} p<0.05$; Fig. $\left.5 E, F\right)$, thus further indicating that the action of Pin1 on PSD-95 is critical for this effect.

Pin 1 regulates the number and the morphology of dendritic spines

NMDARs have been reported to play a key role in dendritic spines formation and morphology (Lamprecht and LeDoux, 2004; Rao and Finkbeiner, 2007). Because in the absence of Pin 1 NMDARs appear enriched at PSD, we tested whether this effect may impact on dendritic spines dynamics. Spines number and morphology were evaluated in Golgi-stained CA1 pyramidal neurons from both mouse genotypes (Fig. 6A). Compared with control, neurons from Pin $1^{-/-}$mice showed a significant in- crease in spines density along stratum radiatum apical dendrites, leading to a rightward shift of the cumulative distribution curve of spine density per dendritic segment compared with Pin $1^{+/+}$ mice (Fig. 6B, left). A significantly larger proportion of mushroom spines was observed in Pin $1^{-/-}$as demonstrated by the respective cumulative frequency plots (Fig. $6 B$, right), whereas the proportion of thin spines did not significantly differ (data not shown). A similar increase in spine density was observed in the hippocampus of Thy-1GFP mice treated with PiB, the pharmacological inhibitor of Pin1 catalytic activity, compared with neurons treated with vehicle (Fig. 6C).

To evaluate whether the increased spines density in Pin $1^{-/-}$ neurons is associated with an enhanced recruitment of NMDARs clusters at synapses, immunocytochemical experiments were performed on cultured hippocampal neurons colabeled for PSD95, GluN1, and VGLUT1, a presynaptic marker of glutamatergic innervation. As shown in Figure 6D, a significant increase in the number of GluN1 colocalized with PSD-95 and juxtaposed to VGLUT1 clusters was detected in Pin $1^{-1-}$ mice respect to controls $\left(41.2 \pm 5.4 \%\right.$ and $63.1 \pm 6.5 \%$, Pin $1^{+/+}$and Pin $1^{-/-}$neurons, respectively). Also in this case, $\mathrm{PiB}$ treatment mimicked the increased in synaptic PSD-95/NMDAR colocalized clusters (Fig. $6 E ; 24.8 \pm 4.3 \%$ and $47.8 \pm 5.7 \%$, DMSO and PiB-treated, respectively). Altogether, these data suggest that Pin 1 negatively regulates the number of spines and NMDAR recruitment at excitatory synapses. 
A

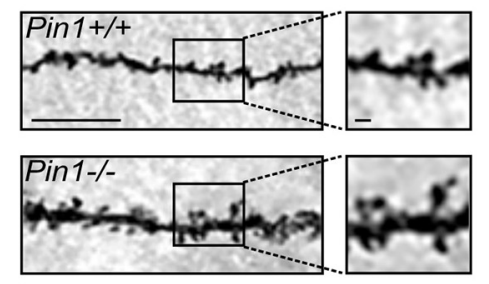

C
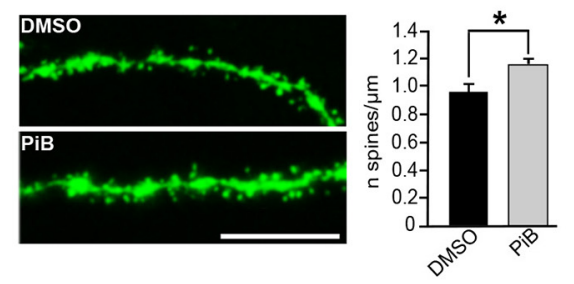

B

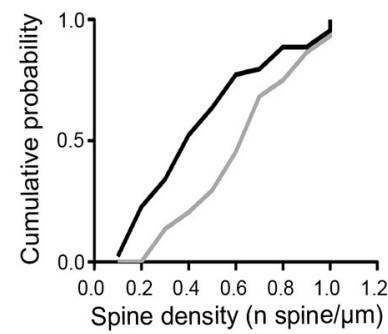

D
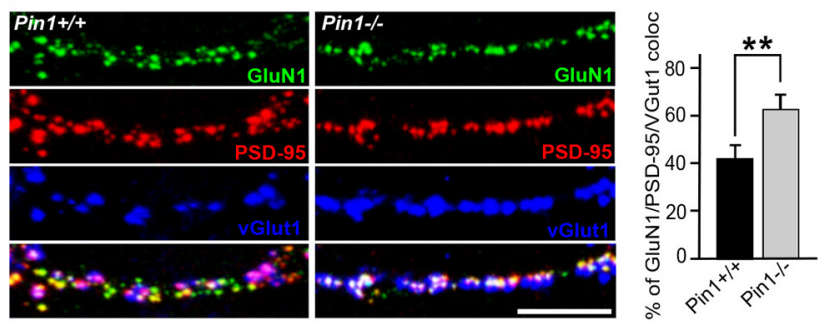

E
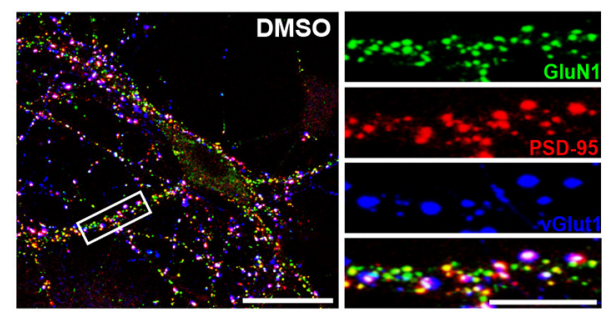

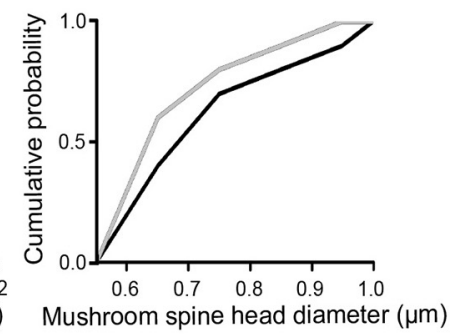

Mushroom spine head diameter $(\mu \mathrm{m})$
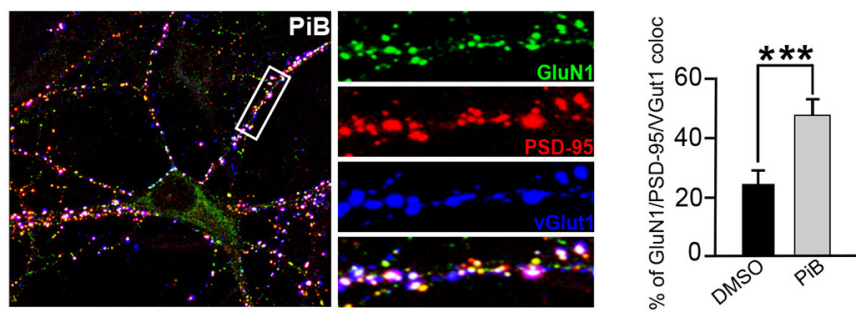

Figure 6. Pin1 regulates spine number and size. $A$, Representative segments of Golgi-stained CA1 pyramidal neurons dendrites. Scale bar, $10 \mu \mathrm{m}$. Selected area are reported on the right. Scale bar, $1 \mu \mathrm{m}$. B. Cumulative probability plots of spine density values (left) measured along $20 \mu \mathrm{m}$ dendritic segments of apical CA1 dendrites in Pin ${ }^{+/+}$(black line) and Pin ${ }^{-/-}$(gray line) mice $(n=$ 5 mice for each genotype; $8-9$ segments for each mouse; ${ }^{*} p=0.003$ Kolmogorov-Smirnov (KS) test). Right, The cumulative probability plots for head width of mushroom spines ( $n=5$ mice for each genotype; 1016 spines; ${ }^{*} p<0.05$, KS test). C, Representative segments of GFP-expressing neurons derived from mice stereotaxically injected with PiB or vehicle (DMSO alone) in the hippocampus. Scale bar, $10 \mu \mathrm{m}$. On the right distribution histogram of spine density along CA 1 apical dendrites of DMSO or PiB-injected animals $\left(n=3 ;\right.$ one-way ANOVA, $\left.F_{(1,27)}=4.77 ;{ }^{*} p=0.03\right)$. D, Example of hippocampal neurons from Pin $1^{+/+}$and Pin $1^{-/-}$immunolabeled for endogenous PSD-95 (red), GluN1 (green), and vGlut1 (blue). Right, Distribution histograms of the percentage of clusters colabeled with PSD-95, GluN1, and VGLUT1. Postsynaptic clustering is demonstrated by apposition of PSD-95/GluN1 clusters to vGlut1-positive terminals on the merge window. Scale bar, $5 \mu \mathrm{m}$. The number of hippocampal neurons investigated in each experiment ( 3 independent experiments) was as follows: $n=10$ for Pin ${ }^{+/+}, n=13$ for Pin $1^{-/-}$; ${ }^{* *} p=0.00057$, Student's $t$ test. $\boldsymbol{E}$, Representative examples of hippocampal neurons treated for $48 \mathrm{~h}$ with PiB or vehicle and immunolabeled as described in $\boldsymbol{D}$. Enlarged boxed areas (scale bar, $5 \mu \mathrm{m}$ ) are shown aside to the corresponding full view image (scale bars, $20 \mu \mathrm{m}$ ). The number of hippocampal neurons investigated in each experiment ( 3 independent experiments) was as follows: $n=11$ for PiB and $n=10$ for DMSO. For each neurons, at least five dendritic regions-of-interest were measured; mean values $\pm S D$, ${ }^{* * *} p<0.0001$, Student's $t$ test).

\section{Discussion}

Here we provide evidence that PSD-95 represents a newly identified target of Pin1-dependent signaling cascade. This scaffold molecule becomes competent to recruit the rotamase upon phosphorylation at specific consensus motifs localized just before the third PDZ domain. Pin 1 activity impacts on the ability of PSD-95 to interact with NMDARs. In particular, Pin1 negatively influences PSD-95/GluN2B complex formation, an effect associated with a decrease in spine density and NMDAR-mediated synaptic transmission.

It is well established that PSD-95 exerts a tight control on postsynaptic maturation and synaptic transmission (El-Husseini et al., 2000; Elias et al., 2006) and its regulated phosphorylation represents one of the key mechanism controlling synaptic targeting and clustering. Phosphorylation can either facilitate PSD-95 delivery to the synapse via association with motor protein complexes or increase its stability at synapses via interaction with other PSD components, including NMDARs. PSD-95 is a recognized target of proline-directed phosphorylation: while cyclindependent kinase 5 phosphorylates Thr19/Pro and Ser25/Pro
$\mathrm{N}$-terminal motifs leading to a downregulation of PSD-95 and NMDARs at synapses (Morabito et al., 2004), JKN1 heavily modify Ser295/Pro site, thus promoting PSD-95 synaptic targeting (Kim et al., 2007). All these phosphorylated forms of PSD-95 are enriched at postsynaptic densities, underlying the importance of this mechanism in controlling the amount of synaptic PSD-95.

The data herein reported not only confirm PSD-95 as a substrate of proline-directed phosphorylation but also unveil that specific post-translational events foster Pin 1 recruitment. This enzyme was found in complex with PSD-95 in brain extracts or upon its ectopic expression in HEK293T cells and its recruitment was entirely dependent on PSD-95 phosphorylation. Several lines of evidence support this notion: (1) the fraction of PSD-95 pulled down by GST-Pin 1 is recognized by the MPM-2 antibody; (2) PSD-95, stripped of all its phosphate groups by phosphatase treatment, is not longer able to bind GST-Pin1; and (3) Pin1Y23A, a mutant impaired in phosphoproteins binding, totally fails to interact with PSD-95 in pull-down and coimmunoprecipitation experiments. 
PSD-95 possesses six potential Pin1 consensus motifs clustered at the N-terminus and at the linker region connecting PDZ2 to PDZ3. Our study points to the second cluster for the phosphorylation-dependent recruitment of Pin1 by PSD-95. Indeed, only the removal of the domain encompassing Ser287 to Ser295, or the sequential disruption of each Pin 1 consensus motif here located, drastically abolished Pin1/PSD-95 interaction. The $\mathrm{N}$-terminal deletion mutant was instead completely ineffective either alone or in conjunction with the linker deletion mutant. The close proximity of these sites to PDZ domains known to be involved in GluN2A/B and NL1 interactions raises the possibility that a conformational shift in this region affects the PDZ binding affinity for the corresponding interactors. Our data demonstrate that Pin1-driven conformational rearrangements mainly impacts on PDZ2, the domain involved in NMDAR recruitment. In coimmunoprecipitation experiments from hippocampus and brain extracts, we consistently detected an enhanced GluN2B/PSD-95 complex formation in the absence of Pin 1 expression, whereas PSD-95/NL1 interaction was quantitatively similar in both genotypes. We also found an overall increase in the amount of GluN2B and GluN1 receptor subunits in synaptosomal preparations derived from Pin $1^{-/-}$hippocampal tissues compared with Pin $1^{+/+}$, further emphasizing the role of Pin 1 as negative modulators of PSD-95/NMDAR interaction. Unexpectedly, PSD-95 total protein levels did not parallel the NMDARs increase. A plausible interpretation of this apparent discrepancy may rely on the fact that PSD-95 at synapses is present in large excess compared with glutamate receptors (Chen et al., 2005). Therefore, just by increasing the fraction of PSD-95 undergoing prolylisomerization can render PSD-95-based scaffold more efficient in trapping NMDARs at postsynapses. These results were validated by immunocytochemical experiments, where a higher number of synaptic PSD-95/GluN1 colabeled clusters was detected in hippocampal neurons from Pin $1^{-/-}$mice, as well as in Pin1expressing neurons treated with $\mathrm{PiB}$, the pharmacological inhibitor of Pin 1 isomerase activity.

In addition electrophysiological data demonstrated an enhanced NMDAR-mediated synaptic transmission in the absence of Pin1 expression. Notably, a similar increase in NMDARmediated currents was uncovered upon ectopic expression of GFP-PSD-95 mutant unable to undergo prolyl-isomerization, indicating that Pin 1 action on PSD-95 represent a key event in boosting its ability to trap NMDARs at synapses. We can exclude that modifications in NMDAR subunit composition (Monyer et al., 1991) contribute to the observed effect because no significant changes in the kinetics of NMDA-mediated synaptic responses or in the ifenprodil-induced depression of synaptic currents emerged between two genotypes.

In line with these results, a higher amount of dendritic spines was unveiled in Golgi-stained pyramidal neurons lacking Pin1 expression. A similar phenotype was observed on hippocampal neurons from Thy-1GFP mice treated with intrahippocampal injection of $\mathrm{PiB}$, indicating that the observed effect depends on the rotamase activity of Pin 1 and not on possible compensatory mechanisms that the embryonic absence of Pin 1 could elicit.

It is interesting to note that the observed increase in spine density in Pin $1^{-/-}$mice is due to a selective gain in mushroom spines, being thin spines content similar in both genotypes. Thin spines are regarded as transient, learning spines, highly dynamic and plastic, that can rapidly form or disappear in response to different levels of synaptic activity (Sala and Segal, 2014). Mushroom spines, instead, are more stable structures, characterized by larger PSDs, accommodating a higher number of glutamate re- ceptors as well as organelles required to sustain local protein synthesis (Hering and Sheng, 2001). It worth noting that the conversion of "learning spines" into "memory spines" (Bourne and Harris, 2007) strengthens activity-dependent synaptic plasticity processes such as LTP, a phenomenon known to be controlled by Pin 1 activity (Westmark et al., 2010). Indeed, Pin 1 has emerged to negatively regulate the induction of dendritic translation required for late LTP maintenance. By combining those evidences with our data, it is tempting to speculate that Pin 1 may affect plasticity not only by regulating de novo protein synthesis necessary to express it, but also by determining the amount of NMDARs initiating plasticity via PSD-95 prolyl-isomerization.

In conclusion, Pin 1 has clearly emerged as key regulator of synaptic transmission. Previous studies identified gephyrin, the core scaffold at GABAergic synapses, and NL2 as the functional transducers of this post-translational mechanism to achieve changes in inhibitory transmission (Zita et al., 2007; Antonelli et al., 2014). The uncovered function of Pin 1 as modulator of PSD95/NMDAR interaction not only strengthen its pivotal contribution in modulating synaptic signaling but also assign to Pin 1 a role in the crosstalk between excitatory and inhibitory transmission, which is of fundamental importance to modulate network activity.

\section{References}

Antonelli R, Pizzarelli R, Pedroni A, Fritschy JM, Del Sal G, Cherubini E, Zacchi P (2014) Pin1-dependent signalling negatively affects GABAergic transmission by modulating neuroligin2/gephyrin interaction. Nat Commun 5:5066. CrossRef Medline

Bourne J, Harris KM (2007) Do thin spines learn to be mushroom spines that remember? Curr Opin Neurobiol 17:381-386. CrossRef Medline

Chen X, Vinade L, Leapman RD, Petersen JD, Nakagawa T, Phillips TM, Sheng M, Reese TS (2005) Mass of the postsynaptic density and enumeration of three key molecules. Proc Natl Acad Sci U S A 102:1155111556. CrossRef Medline

Craven SE, El-Husseini AE, Bredt DS (1999) Synaptic targeting of the postsynaptic density protein PSD-95 mediated by lipid and protein motifs. Neuron 22:497-509. CrossRef Medline

Davis FM, Tsao TY, Fowler SK, Rao PN (1983) Monoclonal antibodies to mitotic cells. Proc Natl Acad Sci U S A 80:2926-2930. CrossRef Medline

Du CP, Gao J, Tai JM, Liu Y, Qi J, Wang W, Hou XY (2009) Increased tyrosine phosphorylation of PSD-95 by Src family kinases after brain ischaemia. Biochem J 417:277-285. CrossRef Medline

El-Husseini AE, Schnell E, Chetkovich DM, Nicoll RA, Bredt DS (2000) PSD-95 involvement in maturation of excitatory synapses. Science 290: 1364-1368. CrossRef Medline

Elias GM, Nicoll RA (2007) Synaptic trafficking of glutamate receptors by MAGUK scaffolding proteins. Trends Cell Biol 17:343-352. CrossRef Medline

Elias GM, Funke L, Stein V, Grant SG, Bredt DS, Nicoll RA (2006) Synapsespecific and developmentally regulated targeting of AMPA receptors by a family of MAGUK scaffolding proteins. Neuron 52:307-320. CrossRef Medline

Etherton M, Földy C, Sharma M, Tabuchi K, Liu X, Shamloo M, Malenka RC, Südhof TC (2011) Autism-linked neuroligin-3 R451C mutation differentially alters hippocampal and cortical synaptic function. Proc Natl Acad Sci U S A 108:13764-13769. CrossRef Medline

Gardoni F, Polli F, Cattabeni F, Di Luca M (2006) Calcium-calmodulindependent protein kinase II phosphorylation modulates PSD-95 binding to NMDA receptors. Eur J Neurosci 24:2694-2704. CrossRef Medline

Gasparini S, Saviane C, Voronin LL, Cherubini E (2000) Silent synapses in the developing hippocampus: lack of functional AMPA receptors or low probability of glutamate release. Proc Natl Acad Sci U S A 97:9741-9746. CrossRef Medline

Gibb R, Kolb B (1998) A method for vibratome sectioning of Golgi-Cox stained whole rat brain. J Neurosci Methods 79:1-4. CrossRef Medline

Hering H, Sheng M (2001) Dentritic spines: structure, dynamics and regulation. Nat Rev Neurosci 2:880-888. CrossRef Medline

Irie M, Hata Y, Takeuchi M, Ichtchenko K, Toyoda A, Hirao K, Takai Y, 
Rosahl TW, Südhof TC (1997) Binding of neuroligins to PSD-95. Science 277:1511-1515. CrossRef Medline

Kim E, Sheng M (2004) PDZ domain proteins of synapses. Nat Rev Neurosci 5:771-781. CrossRef Medline

Kim MJ, Futai K, Jo J, Hayashi Y, Cho K, Sheng M (2007) Synaptic accumulation of PSD-95 and synaptic function regulated by phosphorylation of serine-295 of PSD-95. Neuron 56:488-502. CrossRef Medline

Kornau HC, Schenker LT, Kennedy MB, Seeburg PH (1995) Domain interaction between NMDA receptor subunits and the postsynaptic density protein PSD-95. Science 269:1737-1740. CrossRef Medline

Lamprecht R, LeDoux J (2004) Structural plasticity and memory. Nat Rev Neurosci 5:45-54. CrossRef Medline

Liou YC, Zhou XZ, Lu KP (2011) Prolyl isomerase Pin1 as a molecular switch to determine the fate of phosphoproteins. Trends Biochem Sci 36:501-514. CrossRef Medline

Lu KP, Zhou XZ (2007) The prolyl isomerase PIN1: a pivotal new twist in phosphorylation signaling and disease. Nat Rev Mol Cell Biol 8:904-916. CrossRef Medline

Lu PJ, Zhou XZ, Shen M, Lu KP (1999) Function of WW domains as phosphoserine- or phosphothreonine-binding modules. Science 283: 1325-1328. CrossRef Medline

Manganaro L, Lusic M, Gutierrez MI, Cereseto A, Del Sal G, Giacca M (2010) Concerted action of cellular JNK and Pin1 restricts HIV-1 genome integration to activated CD4+ T lymphocytes. Nat Med 16:329-333. CrossRef Medline

Middei S, Spalloni A, Longone P, Pittenger C, O’Mara SM, Marie H, AmmassariTeule M (2012) CREB selectively controls learning-induced structural remodeling of neurons. Learn Mem 19:330-336. CrossRef Medline

Monyer H, Seeburg PH, Wisden W (1991) Glutamate-operated channels: developmentally early and mature forms arise by alternative splicing. Neuron 6:799-810. CrossRef Medline

Morabito MA, Sheng M, Tsai LH (2004) Cyclin-dependent kinase 5 phosphorylates the $\mathrm{N}$-terminal domain of the postsynaptic density protein PSD-95 in neurons. J Neurosci 24:865-876. CrossRef Medline

Niethammer M, Kim E, Sheng M (1996) Interaction between the C-terminus of NMDA receptor subunits and multiple members of the PSD-95 family of membrane-associated guanylate kinases. J Neurosci 16:2157-2163. Medline

Opazo P, Sainlos M, Choquet D (2012) Regulation of AMPA receptor surface diffusion by PSD-95 slots. Curr Opin Neurobiol 22:453-460. CrossRef Medline

Paoletti P, Bellone C, Zhou Q (2013) NMDA receptor subunit diversity: impact on receptor properties, synaptic plasticity and disease. Nat Rev Neurosci 14:383-400. CrossRef Medline

Poulopoulos A, Aramuni G, Meyer G, Soykan T, Hoon M, Papadopoulos T, Zhang M, Paarmann I, Fuchs C, Harvey K, Jedlicka P, Schwarzacher SW, Betz H, Harvey RJ, Brose N, Zhang W, Varoqueaux F (2009) Neuroligin2 drives postsynaptic assembly at perisomatic inhibitory synapses through gephyrin and collybistin. Neuron 63:628-642. CrossRef Medline

Prybylowski K, Fu Z, Losi G, Hawkins LM, Luo J, Chang K, Wenthold RJ, Vicini S (2002) Relationship between availability of NMDA receptor subunits and their expression at the synapse. J Neurosci 22:8902-8910. Medline

Rao A, Kim E, Sheng M, Craig AM (1998) Heterogeneity in the molecular composition of excitatory postsynaptic sites during development of hippocampal neurons in culture. J Neurosci 18:1217-1229. Medline

Rao VR, Finkbeiner S (2007) NMDA and AMPA receptors: old channels, new tricks. Trends Neurosci 30:284-291. CrossRef Medline

Sala C, Segal M (2014) Dendritic spines: the locus of structural and functional plasticity. Physiol Rev 94:141-188. CrossRef Medline

Vicini S, Wang JF, Li JH, Zhu WJ, Wang YH, Luo JH, Wolfe BB, Grayson DR (1998) Functional and pharmacological differences between recombinant $N$-methyl-Daspartate receptors. J Neurophysiol 79:555-566. Medline

Westmark PR, Westmark CJ, Wang S, Levenson J, O’Riordan KJ, Burger C, Malter JS (2010) Pin1 and PKMzeta sequentially control dendritic protein synthesis. Sci Signal 3:ra18. CrossRef Medline

Williams K (1993) Ifenprodil discriminates subtypes of the N-methyl-Daspartate receptor: selectivity and mechanisms at recombinant heteromeric receptors. Mol Pharmacol 44:851-859. Medline

$\mathrm{Xu}$ W (2011) PSD-95-like membrane associated guanylate kinases (PSDMAGUKs) and synaptic plasticity. Curr Opin Neurobiol 21:306-312. CrossRef Medline

Yoon WJ, Cho YD, Kim WJ, Bae HS, Islam R, Woo KM, Baek JH, Bae SC, Ryoo HM (2014) Prolyl isomerase Pin1-mediated conformational change and subnuclear focal accumulation of Runx2 are crucial for fibroblast growth factor 2 (FGF2)-induced osteoblast differentiation. J Biol Chem 289:8828-8838. CrossRef Medline

Zacchi P, Gostissa M, Uchida T, Salvagno C, Avolio F, Volinia S, Ronai Z, Blandino G, Schneider C, Del Sal G (2002) The prolyl isomerase Pin1 reveals a mechanism to control p53 functions after genotoxic insults. Nature 419:853-857. CrossRef Medline

Zita MM, Marchionni I, Bottos E, Righi M, Del Sal G, Cherubini E, Zacchi P (2007) Post-phosphorylation prolyl isomerization of gephyrin represents a mechanism to modulate glycine receptors function. EMBO J 26 : 1761-1771. CrossRef Medline 